\title{
Dank den Referenten
}

Die Herausgeber und wohl auch fast alle Autoren bedanken sich sehr herzlich bei den Gutachtern, die einen Teil ihrer Arbeitskraft für das Zustandekommen der Beiträge in dieser Zeitschrift, so wie sie sind bzw. nicht sind, investiert haben. Während der Entstehungszeit von Band 3 haben referiert:

G. Altrogge, Hamburg

A. Bachem, Erlangen-Nürnberg

H. H. Bock, Aachen

W. Büler, Dortmund

J. Dathe, Ottobrunn

W. Deutler, Mannheim

W. Dinkelbach, Saarbrücken

v. Dobschütz, Duisseldorf

W. Domschke, Hamburg

A. Drexl, Hamburg

U. Eckhardt, Hamburg

G. Fandel, Hagen

M. Feilmeier, Braunschweig

F. Fersch1, München

D. Fischer, Ottobrunn

W. Gaede, München

M. Gattinger, Erlangen-Nürnberg

H. Glashoff, Hamburg

J. Griese, Dortmund
H.-O. Günther, Berlin

W. Hardeck, Erlangen

$\mathrm{K}$. Hinderer, Karlsruhe

E. Huberty, Dortmund

G. Hübner, Hamburg

Th. Jongen, Enschede

D. Kalin, Bonn

K.-P. Kistner, Bielefeld

J. Kloock, Köln

A. Kolen, Amsterdam

H. Kossbiel, Hamburg

W. Krabs, Darmstadt

J. Lenstra, Amsterdam

K. Lüder, Hamburg

P. Mertens, Erlangen-Nürnberg

M. Meyer, Erlangen-Nürnberg

H. Müller-Merbach, Darmstadt

D. Ohse, Frankfurt

U. Rieder, Ulm
B. Rudolph, Frankfurt

W. Popp, Bern

A.-W. Scheer, Saarbrücken

H. Schellhaas, Darmstadt

Ch. Schneeweiß, Mannheim

R. Schrader, Bonn

Th. M. Schünemann, Hamburg

K.P. Schuster, Jesteburg

J. Sprekels, Hamburg

V. Steinecke, Dortmund

D. Steinhausen, Münster

G. Wenzel, Erlangen-Nürnberg

J. Wessels, Eindhoven

W. Wetterling, Enschede

P.-Th. Wilrich, Berlin

Th. Witte, Osnabrück

B. Wiezorke, Ratingen

U. Zimmermann, Köln

\section{Erratum}

Algorithms for the State Probabilities and Waiting Times in Single Server Queueing Systems with Random and Quasirandom Input and Phase-Type Service Times

H. C. Tijms and M. H. van Hoorn

OR Spektrum 2, 135-152(1981)

Formula (4.5) should be:

$\mathrm{A}_{n, n, i}=\left(\mu+\lambda_{n}\right)^{-1}\left\{1+\mu \mathrm{A}_{n, n, i-1}\right\}, \quad 1 \leqslant i \leqslant r$,

while in formula (4.6) $1 \leqslant k \leqslant n$ should be $1 \leqslant k \leqslant n-1$. 